\title{
Propiedades Microestructurales y Ópticas de Películas Biodegradables a Base de Almidón Termoplástico y Poli ( $\varepsilon$-Caprolactona) con Actividad Antioxidante
}

\author{
Nicolás Piñeros-Guerrero ${ }^{(1,2)}$, Franklin David Sierra-Barahona(1), Yineth Piñeros-Castro(1) y Rodrigo Ortega-Toro(3)* \\ (1) Facultad de Ciencias Naturales e Ingeniería, Programa Ingeniería Química, Universidad Jorge Tadeo Lozano, \\ Carrera 4 \# 22-6, Bogotá D.C. Colombia. (e-mail: nicolasd.pinerosg@utadeo.edu.co; yineth.pineros@utadeo.edu.co) \\ (2) Facultad de Ingeniería, Programa de Maestría en Ingeniería Química, Universidad de Cartagena, Avenida del \\ Consulado Calle 30 No. 48 152, Cartagena de Indias, Colombia. \\ (3) Facultad de Ingeniería, Programa de Ingeniería de Alimentos, Universidad de Cartagena, Avenida del Consulado \\ Calle 30 No. 48 152, Cartagena de Indias, Colombia. (e-mail: rortegap1@unicartagena.edu.co)
}

* Autor a quien debe ser dirigida la correspondencia.

Recibido Nov. 30, 2018; Aceptado Feb. 5, 2019; Versión final Feb. 27, 2019, Publicado Ago. 2019

\section{Resumen}

Se ha estudiado el efecto de la adición de un extracto acuoso rico en fenoles, provenientes de cascarilla de arroz, sobre las propiedades microestructurales y ópticas de películas biodegradables a base de almidón termoplástico y poli ( $\varepsilon$-caprolactona). Para el extracto de cascarilla de arroz se determinó la cantidad de sólidos solubles, el contenido de compuestos fenólicos y la actividad antioxidante. En el caso de las películas, se estudió su morfología mediante Microscopía Electrónica de Barrido y se determinó la transmitancia interna, color y brillo. Los resultados muestran diferencias estadísticamente significativas $(p<0.05)$ para la transmitancia, color y brillo del material. En cuanto a su morfología, la adición de fenoles generó películas menos rugosas, más homogéneas y compactas. Basado en los resultados del trabajo, se concluye que la adición del extracto afecta significativamente la estructura del material modificando sus propiedades ópticas.

\section{Microstructural and Optical Properties of Biodegradable Films Based on Thermoplastic Starch and Poly ( $\varepsilon$-Caprolactone) with Antioxidant Activity}

\begin{abstract}
The effect of the addition of an extract rich in phenols, from rice husks, on the microstructural and optical properties of biodegradable films based on thermoplastic starch and poly ( $\varepsilon$-caprolactone) has been studied. For the rice husk extract, the amount of soluble solid, the content of phenolic compounds and the antioxidant activity were determined. In the case of films, their morphology was studied by Scanning Electron Microscopy and their internal transmittance, colour and brightness were determined. The results show statistically significant differences $(p<0.05)$ for the transmittance, colour and brightness of the material. Regarding its morphology, the addition of phenols generated less rough, and more homogeneous and compact films. Based on the results of the work, it is concluded that the addition of extract significantly affects the structure of the material by modifying its optical properties.
\end{abstract}




\section{INTRODUCCIÓN}

En los últimos años, los materiales biodegradables han sido ampliamente estudiados debido a la necesidad de sustituir los polímeros convencionales no biodegradables, ya que éstos generan una enorme problemática ambiental, por lo cual es necesario explorar nuevos materiales amigables con el medio ambiente y con capacidad biodegradable. Entre estos materiales, se han estudiado películas activas biodegradables a base de almidón para aplicación en sistemas de envasado de alimentos por medio de dos métodos de obtención, uno de ellos denominado vertido el cual consiste en disolver un polímero en un solvente orgánico y luego el vertimiento en un molde en donde mediante evaporación del disolvente la mezcla alcanza la consistencia de un plástico. El otro método es conocido como termoprocesado donde el polímero es fundido o ablandado por procesos térmicos y moldeado por distintas técnicas tales como moldeo por compresión, soplado, inyección y extrusión, esta última es la metodología más utilizada en los procesos industriales.

El almidón termoplástico (TPS) es almidón que ha sido modificado por la adición de plastificantes como glicerina, agua, sorbitol entre otros y, procesado bajo condiciones de presión y temperatura hasta cambiar la estructura cristalina nativa del almidón, generándose un almidón termoplástico amorfo, el TPS es ambientalmente amigable debido a que proviene de fuentes renovables y puede incorporarse al suelo como abono orgánico (Villada et al., 2008). Este material frente a los plásticos convencionales presenta desventajas como solubilidad en agua, alta higroscopicidad, envejecimiento rápido debido a la retrogradación y limitadas propiedades mecánicas lo cual afecta algunas aplicaciones tales como empaques (Luna et al., 2010). Estos problemas se han resuelto por medio de la mezcla en bajas proporciones de otros polímeros y aditivos naturales que mejoran estas propiedades, como es el caso de almidón termoplástico con baja proporción de poli ( $\varepsilon$-caprolactona) esta mezcla genera películas más elásticas y estables, con una menor permeabilidad al vapor de agua. Por otro lado, la adición de aditivos naturales mejora y agrega propiedades al material. Talón et al., (2017) reportaron que la adición de un extracto de tomillo rico en fenoles a una matriz de almidón termoplástico con quitosano generó cambios en el color obteniendo un polímero más oscuro, más rojizo y menos transparente, las películas presentaron algunos cambios en la microestructura, mejorando las propiedades físicas y mecánicas, además este material contiene capacidad antioxidante. En un estudio homólogo, Carissimi et al., (2018) adicionaron un extracto de micro algas a una matriz de almidón de yuca, las películas con el extracto presentaron un incremento en la elongación de la película, esfuerzo a la ruptura y disminuyeron el esfuerzo a la tensión y el módulo de Young; éstas películas fueron usadas como un recubrimiento de salmón, lo cual disminuyó la pérdida de humedad y retraso la oxidación lipídica en el pescado.

Los componentes antioxidantes naturales han sido usados en diferentes mezclas de polímeros con la finalidad de mejorar sus propiedades, Pastor et al. (2013) adicionaron el antioxidante natural resveratrol presente en diferentes variedades de plantas a dos diferentes polímeros compuestos principalmente por quitosano y metilcelulosa, la adición de este componente generó cambios estructurales en las matrices, generando películas menos deformables y resistentes a la fractura; en cuanto a las propiedades ópticas se obtuvieron películas más opacas y menos brillantes aunque poseían actividad antioxidante. El $\beta$-caroteno, uno de los carotenoides con actividad antioxidante más abundantes en la naturaleza, se empleó en una matriz compuesta por tres diferentes polímeros ácido poliláctico (PLA), poli ( $\varepsilon$-caprolactona) $(\mathrm{PCL})$ y polihidroxibutirato (PHBV); la adición del $\beta$-caroteno dio como resultado incrementos significativos en la deformación a la ruptura y menor módulo de Young (López-Rubio y Lagaron, 2010). Tran et al.,(2017)reportó la incorporación de polvo de remolacha roja en bioelastomeros basados en almidón con el fin de obtener biocompuestos flexibles con propiedades antioxidantes, los bioelastomeros eran hidrofóbicos y resistentes a la disolución en agua. El polvo de remolacha aumentó el módulo de Young de los bioelastomeros sin comprometer su capacidad de elongación. El color en los envases es una propiedad muy importante a la hora de atraer el interés de los consumidores. El uso de carotenoides como colorantes para materiales poliméricos representa una forma ecológica de obtener envases de colores. Stoll et al.,(2018) reportaron la incorporacion de extractos carotenoides en una matriz de acido polilactico (PLA), los extractos redujeron la transparencia de las películas siendo la bixina el carotenoide mas estable en condiciones de oscuridad y luz. Boom et al. (2018) evaluaron la actividad antioxidante de aceites ensenciales obtenidos de eucalipto y determinaron que estos aceites contienen fenoles totales entre $(600-950 \mathrm{mg} / \mathrm{L})$ y una actividad antioxidante de $440-600 \mu \mathrm{mol} / \mathrm{L}$.

La modificación química, física y enzimática son un recurso empleado para mejorar propiedades especificas de los materiales basados en almidón. El entrecruzamiento es un tipo de modificación química aplicada al almidon, donde se refuerzan los enlaces de hidrogeno de las moleculas de almidon mejorando la resistencia mecanica, solubilidad y la estabilidad termica (Garavand et al., 2017). La oxidacion es un mecanismo de modificacion quimica empleado para modificar el almidon generando una alternancia estructural, reduciendo la viscosidad y generando nuevos grupos funcionales como carbonilo y carboxilo, existen muchos tipos de agentes oxidantes como el hipoclorito de sodio, ácidos orgánicos (ácido acético, ácido cítrico y ácido láctico), permanganato de magnesio, cromo y peróxido de hidrógeno, este último es uno de los agentes oxidantes utilizados para preparar almidón oxidado (Tanetrungroj y Prachayawarakorn, 2018). Uno de los metodos de 
modificacion fisica de almidon es el tratamiento de calor-humedad, esta metodologia consiste en calentar los granulos de almidon a una temperatura superior a la transicion vitrea y por debajo de la temperatura de gelatinizacion durante un peridodo de tiempo generalmente de 16 horas y manteniendo un rango de humedad inferiror al 35\% p/p (Hoover, 2010). Hu et al., (2018) modificaron almidón de papa con vapor sobrecalentado a dos diferentes temperaturas $\left(100-160^{\circ} \mathrm{C}\right)$, se encontró que el tratamiento modificó efectivamente las estructuras moleculares y propiedades fisicoquimicas del almidon sin afectar la estructura granular, el hinchamiento, la solubilidad, transparencia, entre otras se redujeron significativamente y se evidencio el aumento de la temperatura de pegado. Arijaje et al., (2017) estudiaron el efecto de la $\beta$-amilasa en complejos solubles e insolubles de almidon de papa con acido linoleico donde se encontro que el grado de acetilacion fue mayor en los compeljos solubles que en los insolubles, la acetilacion y las temperaturas de fusion maximas para complejos insolubles disminuyeron.

En este contexto, en este trabajo se evaluó el efecto de la adición de un extracto acuoso antioxidante proveniente de la cascarilla de arroz, sobre las propiedades ópticas y microestructurales de un material polimérico a base de almidón de yuca y poli ( $\varepsilon$-caprolactona).

\section{MATERIALES Y MÉTODOS}

En esta sección se describirá el procedimiento seguido para la obtención y caracterización tanto del extracto de cascarilla de arroz rico en antioxidantes como de las películas biodegradables.

\section{Obtención del extracto antioxidante}

En cuanto a la obtención del extracto antioxidante (ANT), se utilizó un reactor de alta presión marca FIQ LTDA con capacidad de $5 \mathrm{~L}$, en este se procesaron $500 \mathrm{~g}$ de cascarilla de arroz previamente molida en un molino de palas marca RESTCH y tamizada en un juego de tamices HUMBOLDT $(0,4$ a $0,5 \mathrm{~mm})$ y se agregaron 4,5 $\mathrm{L}$ de agua destilada. Las condiciones de extracción fueron temperatura de $160^{\circ} \mathrm{C}$ y presión de 10 bares por un periodo de $30 \mathrm{~min}$. Posteriormente se realizó filtración para recuperar el extracto, el cual se mantuvo en condiciones de refrigeración a $4{ }^{\circ} \mathrm{C}$ para su posterior caracterización.

\section{Caracterización del extracto antioxidante}

Para iniciar la caracterización el extracto fue centrifugado al 10000 rpm en una centrifuga EBA 200 durante 10 minutos; para determinar sólidos solubles en el extracto, se tomó una alícuota de $1 \mathrm{~mL}$ del extracto y se colocó en una placa de Petri previamente tarada, se eliminó el agua por evaporación a $50^{\circ} \mathrm{C}$ en un horno de convección forzada OMH 100 por un tiempo de 24h, por diferencia de masa, se obtuvo la concentración de sólidos solubles. La concentración de compuestos fenólicos totales se determinó mediante método espectrofotométrico, utilizando el reactivo Folin-Ciocalteu y la metodología descrita por Singleton (1965). La actividad antioxidante total se estableció mediante el método DPPH (2,2-difenil-1-picrilhidrazina) (BrandWilliams et al., 1995) y se expresó como equivalentes Trolox (ET) en mgET/100 g de cascarilla seca.

\section{Obtención de las películas}

Para la formulación se mezcló almidón, glicerol, agua y/o policaprolactona y/o extracto antioxidante de acuerdo a lo establecido en la Tabla 1. La matriz polimérica de almidón termoplástico (TPS) se obtuvo mediante mezclado en fundido y moldeo por compresión; se cargó la mezcla en una mezcladora de doble rodillo QIAOLIAN GK-50/80 a una temperatura de $160^{\circ} \mathrm{C}$ y $8 \mathrm{rpm}$ durante un tiempo de 30 minutos hasta que se obtuvo una pasta homogénea. La pasta se redujo de tamaño y se almacenó a $53 \%$ de humedad durante 7 días. Las películas se obtuvieron en una prensa hidráulica QIAOLIAN $100 \mathrm{~T}$ a una temperatura de $130{ }^{\circ} \mathrm{C}$ con un tiempo de precalentamiento de 3 minutos, se fundieron durante 2 minutos a $5 \mathrm{MPa}$, se moldearon por 3 minutos a $20 \mathrm{MPa}$, y la etapa de enfriamiento fue de 3 minutos a $5 \mathrm{Mpa}$.

Tabla 1: Fracción másica (Xi, g compuesto / g de película seca) de los diferentes componentes (Almidón: S, Glicerol: Gli, Policaprolactona: PCL, Extracto de antioxidante: ANT).

\begin{tabular}{|l|c|c|c|c|}
\hline Formulación & $S$ & Gli & PCL & ANT \\
\hline TPS & 0,7692 & 0,2308 & - & - \\
\hline TPS-PCL $(10 \%)$ & 0,7143 & 0,2143 & 0,0714 & - \\
\hline TPS-PCL $(10 \%)-A N T(0,1 \%)$ & 0,7138 & 0,2141 & 0,0714 & 0,0007 \\
\hline TPS-ANT $(0,1 \%)$ & 0,7686 & 0,2306 & - & 0,0008 \\
\hline TPS-PCL $(10 \%)-A N T(0,3 \%)$ & 0,7128 & 0,2138 & 0,0713 & 0,0021 \\
\hline TPS-ANT $(0,3 \%)$ & 0,7675 & 0,2302 & - & 0,0023 \\
\hline
\end{tabular}




\section{Microscopia de Barrido Electrónico (SEM)}

El análisis micro estructural de las películas se llevó a cabo usando un microscopio de barrido electrónico JEOL JSM-6490LV, las muestras se acondicionaron en un desecador con P2O5 durante dos semanas para asegurar que no hay presencia de agua en la muestra (humedad relativa teórica en el desecador 0). Posteriormente las películas se congelaron con nitrógeno líquido y se sometieron a un proceso de crio fractura para observar la sección transversal y la superficie de las muestras. Las películas se recubrieron con oro y se observaron con un voltaje de $10 \mathrm{KV}$.

\section{Transmitancia}

La transmitancia de las películas se determinó en un rango de luz UV-VIS a probetas de $(1 \mathrm{~cm} \times 3 \mathrm{~cm})$ que estuvieron acondicionadas a $20^{\circ} \mathrm{C}$ y $53 \% \mathrm{HR}$, usando un espectrofotómetro UV-VIS (Thermo Scientific, Evolution 300), dentro de un rango de longitud de onda de entre 190 a $1100 \mathrm{~nm}$.

\section{Colorimetría}

Se usó análisis colorimétrico para evaluar el color de las películas, se analizó la diferencia total del color para cada muestra considerando como blanco TPS, se empleó el espacio de color (CIE $\left.L^{*} A^{*} B^{\star}\right)$ las coordenadas se midieron con un colorímetro (Konica Minolta modelo CR-400) con el fin de establecer con la norma ISO 12647-2 la calidad de color de cada muestra (ISO 12647-2, 2004).

\section{Medición de brillo}

El brillo se determinó en la superficie de la película, en un ángulo de incidencia de $85^{\circ}$ por medio de un medidor de brillo (Multi Gloss 268, Minolta, Alemania), siguiendo el método estándar ASTM D523. Las mediciones se llevaron a cabo por triplicado en cada película. Los resultados muestran una unidad de brillo (GU) relativa a una superficie altamente pulida de vidrio negro estándar con un valor cercano a 100 GU.

\section{Análisis estadístico}

El análisis de los datos se realizó mediante ANOVA y las medias fueron comparadas con test LSD utilizando el software Statgraphics, a un nivel del 95\% de confianza $(p<0.05)$.

\section{RESULTADOS Y DISCUSIÓN}

A continuación, se describen los resultados obtenidos en el trabajo. Para una mejor presentación y discusión los resultados se presentan en cinco subsecciones: i) Caracterización del extracto antioxidante; (ii) Microscopia de Barrido Electrónico; (iii) Transmitancia; (iv) Colorimetría; y (v) Medición de brillo

\section{Caracterización del extracto antioxidante}

En la Tabla 2 se presentan los valores obtenidos de la caracterización del extracto antioxidante, el extracto presentó una concentración de sólidos solubles de $31,4 \mathrm{~g} / \mathrm{L}$, constituidos principalmente por azúcares y compuestos fenólicos. El contenido de fenoles totales es expresado en mili equivalentes de ácido gálico (mEAG) por cada $100 \mathrm{~g}$ de cascarilla seca y se obtuvo un valor de $12,27 \mathrm{mgEAG} / 100 \mathrm{~g}$ de cascarilla seca.

Tabla 2: Concentración de sólidos solubles, fenoles totales y actividad antioxidante de extracto datos comparativos obtenidos de Huang et al, (2012) y Mesa-vanegas et al, (2010)

\begin{tabular}{|c|c|c|c|c|}
\hline Determinación & $\begin{array}{c}\text { Extracto } \\
\text { cascarilla de } \\
\text { arroz }\end{array}$ & $\begin{array}{c}\text { Extracto cascarilla de } \\
\text { arroz japónica K139 } \\
\text { (Huang) }\end{array}$ & $\begin{array}{c}\text { Extracto } \\
\text { cascarilla de } \\
\text { arroz indica } \\
\text { TS10 (Huang) }\end{array}$ & $\begin{array}{c}\text { Extracto } \\
\text { calophyllum } \\
\text { CBTExtH } \\
\text { (Mesa-vanegas) }\end{array}$ \\
\hline Sólidos solubles $(\mathrm{g} / \mathrm{mL})$ & $0,031 \pm 0,0007$ & - & - & - \\
\hline Fenoles totales $(\mathrm{gEAG} / \mathrm{kg})$ & $0,13 \pm 0,3$ & $0,97 \pm 1$ & $0,71 \pm 1$ & $5,9 \pm 5,5$ \\
\hline Actividad antioxidante $(\mathrm{gET} / \mathrm{kg})$ & $66,92 \pm 0,3$ & - & - & $80,00 \pm 0,63$ \\
\hline
\end{tabular}

El valor obtenido de compuestos fenólicos a partir de la cascarilla de arroz seca, es similar al reportado en materiales de Taiwan (Huang y Ng, 2012). Adicionalmente, se evidencia que el extracto de la cascarilla de arroz tiene alta actividad antioxidante a pesar de su bajo contenido de fenoles totales, cuando se compara 
con otros materiales como el extracto de Calophyllum el cual posee una cantidad alta de compuestos fenólicos, empleando así una menor cantidad de extracto para obtener la misma capacidad antioxidante.

\section{Microscopia de Barrido Electrónico (SEM)}

En la Figura 1 se presentan las micrografías SEM de la sección transversal de las formulaciones estudiadas. Las películas fueron crio fracturadas con nitrógeno líquido y metalizadas con oro, las imágenes fueron tomadas a una resolución $500 \mathrm{x}$. Las películas fueron acondicionadas por un tiempo de 1 semana a $20^{\circ} \mathrm{C}$ y $53 \%$ de humedad relativa.

Las películas de TPS presentan estructura homogénea en comparación a la morfología presentada por las películas que contienen PCL, se observa una distribución aleatoria de PCL en la matriz de almidón para la formulación 2. Sin embargo, para las formulaciones que contienen extracto antioxidante se evidencia que a medida que la concentración de este aumenta, la morfología de la película se ve más homogénea, se disminuye la prominencia de los fragmentos de PCL en la matriz de almidón como se muestra en la micrografía de la formulación 5 en donde se observan un tipo de perforación, filamentos de PCL y una mayor cantidad de área homogénea.

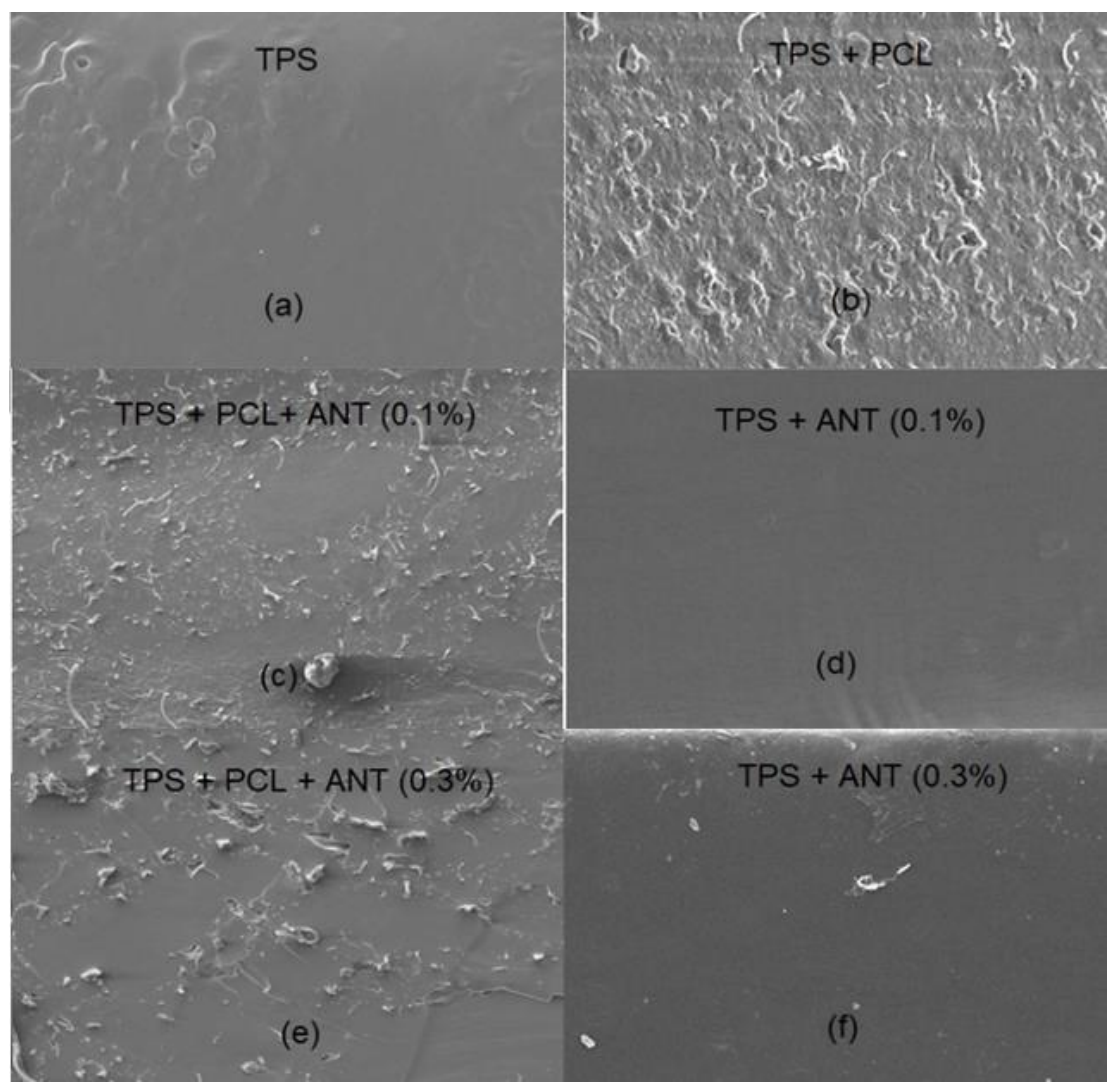

Fig. 1: Micrografía SEM sección transversal de películas a magnitud de 500x.

Las áreas homogéneas que se aprecian en la micrografía de la formulación 5 son efecto de la plastificación que genera el extracto antioxidante a la matriz de polimérica (TPS $+P C L$ ), la fase de PCL se puede distinguir claramente debido a su comportamiento dúctil, que define un patrón particular de fractura criogénica, promoviendo formaciones filamentosas estiradas.

\section{Transmitancia}

La transmitancia interna se define como la fracción de luz que pasa a través de una muestra, como se puede observar tanto a una longitud de onda de $650 \mathrm{~nm}$ como a $750 \mathrm{~nm}$, la incorporación de PCL disminuye considerablemente el porcentaje de luz que pasa sobre los películas que contienen PLC, esto se debe a que la PCL es un polímero opaco mientras que el TPS por tener una estructura lineal es un polímero que se caracteriza por presentar regiones amorfas que permiten el paso de mayor cantidad de luz a través de la película, haciendo de éste un polímero semitransparente. Las películas que se les adiciono PLC (10\%) fueron los que presentaron menos porcentaje de transmitancia en comparación al TPS ya que su estructura es menos amorfa y permite menos paso de luz. La adición de $0.1 \%$ y $0.3 \%$ de antioxidante también logra disminuir 
considerablemente la translucidez de las películas con y sin PCL debido a que el antioxidante presenta un color rojizo que permite que absorba la luz y no permita el paso de esta. Por esta razón las películas que contienen PCL y antioxidante son los que presentan valores más bajos de transmitancia.

En la Tabla 3 se presenta los valores de transmitancia interna a dos diferentes longitudes de onda, estos valores fueron obtenidos de un barrido de 190 a $1100 \mathrm{~nm}$ en donde se midió la transmitancia de las formulaciones estudiadas. Diferentes letras en los superíndices dentro de la misma columna indican diferencias significativas entre las formulaciones $(p<0,05)$. Las formulaciones fueron acondicionadas por un tiempo de 1 semana a $53 \%$ de humedad relativa y $20^{\circ} \mathrm{C}$ antes de tomar las mediciones.

Tabla 3: Valores medios y desviaciones estándar del porcentaje de transmitancia interna

\begin{tabular}{|l|c|c|}
\hline Formulación & $\% \mathrm{Ti}(650 \mathrm{~nm})$ & $\% \mathrm{Ti}(750 \mathrm{~nm})$ \\
\hline TPS & $65 \pm 4^{\mathrm{b}}$ & $67 \pm 4^{\mathrm{b}}$ \\
\hline TPS-PCL (10\%) & $28 \pm 3^{\mathrm{b}}$ & $32 \pm 3^{\mathrm{b}}$ \\
\hline TPS-PCL (10\%)-ANT $(0,1 \%)$ & $11 \pm 2^{\mathrm{a}}$ & $12 \pm 2^{\mathrm{a}}$ \\
\hline TPS-ANT (0,1\%) & $55 \pm 2^{\mathrm{a}}$ & $56 \pm 3^{\mathrm{a}}$ \\
\hline TPS-PCL (10\%)-ANT (0,3\%) & $12 \pm 2^{\mathrm{a}}$ & $13 \pm 2^{\mathrm{a}}$ \\
\hline TPS-ANT $(0,3 \%)$ & $60 \pm 3^{\mathrm{a}}$ & $61 \pm 2^{\mathrm{a}}$ \\
\hline
\end{tabular}

\section{Colorimetría}

En la Tabla 4 se presentan los valores medios y las desviaciones estándar de las coordenadas $\left(L^{*} a^{*} b^{*}\right)$ del espacio de color CIE $L^{*} A^{*} B^{*}$, con las cuales se calcularon los deltas de color con respecto al blanco (TPS). Diferentes letras en los superíndices dentro de la misma columna indican diferencias significativas entre las formulaciones $(p<0.05)$. Las películas fueron acondicionadas por un tiempo de 1 semana a $20^{\circ} \mathrm{C}$ y $53 \%$ de humedad relativa.

Tabla 4: Valores medios y desviaciones estándar de las coordenadas de color

\begin{tabular}{|l|c|c|c|c|c|c|c|}
\hline Formulación & $L^{*}$ & $a^{*}$ & $b^{*}$ & $\Delta L^{*}$ & $\Delta a^{*}$ & $\Delta b^{*}$ & $\Delta E^{*}$ \\
\hline TPS & $74,40 \pm 0,12^{\mathrm{ab}}$ & $7,46 \pm 0,02^{\mathrm{a}}$ & $5,92 \pm 0,03^{\mathrm{b}}$ & - & - & - & - \\
\hline TPS-PCL (10\%) & $75,00 \pm 0,02^{\mathrm{ab}}$ & $7,36 \pm 0,02^{\mathrm{a}}$ & $5,89 \pm 0,02^{\mathrm{b}}$ & 0,61 & $-0,10$ & $-0,03$ & 0,62 \\
\hline TPS-PCL (10\%)-ANT $(0,1 \%)$ & $74,77 \pm 0,14^{\mathrm{b}}$ & $7,22 \pm 0,02^{\mathrm{b}}$ & $4,42 \pm 0,06^{\mathrm{a}}$ & 0,37 & $-0,24$ & $-1,50$ & 1,56 \\
\hline TPS-ANT (0,1\%) & $74,8 \pm 0,2^{\mathrm{b}}$ & $8,19 \pm 0,02^{\mathrm{b}}$ & $3,21 \pm 0,08^{\mathrm{a}}$ & 0,45 & 0,73 & $-2,71$ & 2,84 \\
\hline TPS-PCL (10\%)-ANT (0,3\%) & $74,3 \pm 0,2^{\mathrm{a}}$ & $7,17 \pm 0,03^{\mathrm{ab}}$ & $4,19 \pm 0,10^{\mathrm{a}}$ & $-0,07$ & $-0,29$ & $-1,73$ & 1,75 \\
\hline TPS-ANT $(0,3 \%)$ & $74,49 \pm 0,11^{\mathrm{a}}$ & $8,16 \pm 0,02^{\mathrm{ab}}$ & $3,06 \pm 0,03^{\mathrm{a}}$ & 0,09 & 0,70 & $-2,86$ & 2,95 \\
\hline
\end{tabular}

Se evidencio que la adición del extracto antioxidante genera diferencias significativas $(p<0,05)$ en las coordenadas de color $\left(L^{*}, a^{*} y b^{*}\right)$, esto se debe a el color rojo característico que presenta el extracto antioxidante el cual al ser mezclado con la matriz polimérica genera cambios en el color de las películas, estos cambios fueron cuantificados como la diferencia total de color $\left(\Delta \mathrm{E}^{*}\right)$ y fueron comparados entre formulaciones con y sin PCL tomando como efecto principal la adición de extracto antioxidante, se evidencio que las formulaciones que contienen PCL tienen una buena calidad de color excepto la formulación que no contiene extracto antioxidante, esta presenta una calidad excelente y las que no contienen PCL tienen una calidad normal de color según el estándar (ISO 12647-2, 2004).

\section{Medición de brillo}

En la Tabla 5 se presentan los valores medios y desviaciones estándar de la medición de unidades de brillo (UB) a diferentes películas de cada formulación comparado con un patrón de calibración de cristal pulido negro con un índice de refracción de 100 UB. Diferentes letras en los superíndices dentro de la misma columna indican diferencias significativas entre las formulaciones $(p<0,05)$. Las películas fueron acondicionadas por un tiempo de 1 semana a $20^{\circ} \mathrm{C}$ y $53 \%$ de humedad relativa.

A las formulaciones que se les adiciono PCL presentan menor brillo respecto a las formulaciones que no incluyen este componente en su matriz. 
Tabla 5: Valores medios y desviaciones estándar de brillo de las películas

\begin{tabular}{|l|c|}
\hline Formulación & Brillo (UB) \\
\hline TPS & $38,7 \pm 0,4^{\mathrm{b}}$ \\
\hline TPS-PCL $(10 \%)$ & $16,4 \pm 0,4^{\mathrm{b}}$ \\
\hline TPS-PCL $(10 \%)$-ANT $(0,1 \%)$ & $16,0 \pm 0,2^{\mathrm{c}}$ \\
\hline TPS-ANT $(0,1 \%)$ & $52,9 \pm 1,5^{\mathrm{c}}$ \\
\hline TPS-PCL $(10 \%)$-ANT $(0,3 \%)$ & $6,3 \pm 0,8^{\mathrm{a}}$ \\
\hline TPS-ANT $(0,3 \%)$ & $32,3 \pm 1,5^{\mathrm{a}}$ \\
\hline
\end{tabular}

Este efecto se debe a que la PCL es un polímero opaco, cuando se hace la medición de brillo a las películas con un ángulo de incidencia de $85^{\circ}$ la luz que incide sobre el material no es reflejada en su totalidad, mientras que las formulaciones que no tienen PCL presentan una superficie lisa que permite que la luz que incide sobre el material sea reflejada en su totalidad obteniendo mayor brillo en la película, los valores que se obtienen de brillo van fuertemente ligado con el proceso de termo moldeado de los películas ya que cuando se obtienen más lisas el material es más brillante, por el contrario, cuando se obtienen películas rugosas reflejan menos cantidad de luz.

\section{DISCUSIÓN FINAL}

En la tabla 6 se presentan valores de propiedades ópticas de diferentes investigaciones en las que se añadió un extracto antioxidante a diferentes tipos de materiales, se observó que todos los materiales estudiados en las investigaciones sufrieron un aumento en los valores de transmitancia interna debido a la adicción de los extractos, esto se puede deber a un cambio en la estructura haciéndose esta menos compacta con una índice refracción más bajo, generando un material más brillante como lo asegura Bonilla et al, (2013), este mismo efecto fue observado en las micrografías SEM en donde a medida que la concentración del extracto estudiado aumenta se obtienen películas menos rugosas. En cuanto a la luminosidad, croma y matiz de los materiales en algunos casos son fuertemente afectados por el color natural de los extractos, Carissimi et al, (2018) observo que la adicción de extracto de microalgas genero materiales más oscuros por lo que la transparencia disminuyo y consecuentemente los valores de luminosidad disminuyeron a medida que la concentración de microalgas aumento, la misma tendencia fue observada en la presente investigación.

Pastor et al, (2013) observaron que las películas obtenidas disminuyeron su brillo a medida que aumentó la concentración de resveratrol, esto se puede deber a la presencia de cristales de resveratrol generando una superficie con mayor heterogeneidad, caso contrario al observado en esta investigación donde se observó que el extracto de cascarilla de arroz plastifico los materiales generando una estructura homogénea. Talón et al, (2017) observaron que la adicción de extracto antioxidante de ácido tánico a los materiales provocó cambios significativos, generando un color más oscuro y rojizo, la luminosidad se vio significativamente reducida por la adicción del extracto, también relacionó la tonalidad rojiza con la oxidación de los polifenoles durante el secado enfatizando el efecto protector de la matriz de quitosano contra la oxidación de los polifenoles. De esta manera se observa que los resultados obtenidos están acordes a la literatura internacional y en algunos casos se presentan muchas similitudes. Los materiales desarrollados presentan buenas propiedades ópticas necesarias para el empaque de alimentos y cuentan con capacidad antioxidante estos materiales pueden ser empleados para el empaque de aceites, debido a que los átomos de carbono de los ácidos grasos polinsaturados en presencia de luz y oxigeno atmosférico sufren reacciones de oxidación por radicales libres que confieren al aceite malos olores y sabores.

Tabla 6: Valores medios y desviaciones estándar para transmitancia interna (Ti), luminosidad $\left(L^{*}\right)$, croma $\left(C^{*}\right.$ ab), matiz $\left(h^{*} a b\right)$ y brillo de diferentes materiales compuestos por quitosano $(\mathrm{CH})$, almidón $(\mathrm{S})$, ácido tánico (TA), resveratrol $(\mathrm{R})$, almidón de trigo (WS), a-tocoferol (TP), D. tertiolecta (ED) datos comparativos obtenidos de Talón et al, (2017), Pastor et al, (2013), Bonilla et al, (2013) y Carissimi et al, (2018)

\begin{tabular}{|c|c|c|c|c|c|}
\hline Formulación & $\begin{array}{c}\% \mathrm{Ti}(460 \\
\mathrm{nm})\end{array}$ & $L^{*}$ & $h^{*}{ }_{a b}$ & $C^{*}{ }^{*}{ }$ & Brillo (UB) \\
\hline TPS-ANT (0,3\%) & $52,0 \pm 0,3$ & $74,5 \pm 0,1$ & $21,8 \pm 1,0$ & $8,7 \pm 1,0$ & $32,3 \pm 1,5$ \\
\hline CH:S:TA (Talón) & $53,4 \pm 0,3$ & $56,0 \pm 1,0$ & $78,1 \pm 0,4$ & $31,0 \pm 1,0$ & $28,0 \pm 7,0$ \\
\hline CH + R100 (Pastor) & $60,0 \pm 0,3$ & $81,0 \pm 2,0$ & $90,0 \pm 2,0$ & $17,0 \pm 3,0$ & $36,0 \pm 14$ \\
\hline WS:CH:TP (Bonilla) & $85,0 \pm 0,3$ & $83,0 \pm 0,6$ & $89,5 \pm 0,6$ & $15,7 \pm 0,3$ & $13,0 \pm 3,0$ \\
\hline ED 2.0 (Carissimi) & - & $69,4 \pm 0,3$ & $82,2 \pm 1,0$ & $73,9 \pm 1,0$ & - \\
\hline
\end{tabular}




\section{CONCLUSIONES}

De los resultados mostrados y su discusión se pueden obtener las siguientes conclusiones sobre el efecto del extracto antioxidante proveniente de la cascarilla de arroz y PCL sobre las propiedades ópticas y morfológicas del material:

1) La adición de extracto antioxidante y PCL a la matriz de almidón afecta significativamente las propiedades ópticas de las películas generando un menor brillo y una calidad de color menor.

2) Se observó en las micrografías SEM que la adición de PCL tiene cambios significativos en la morfología obteniéndose películas más rugosas. Sin embargo, la adición de extracto antioxidante plastifica la matriz de TPS con PCL disminuyendo significativamente la rugosidad de las películas.

\section{REFERENCIAS}

Arijaje, E.O. e Y.J. Wang, Effects of chemical and enzymatic modifications on starch-linoleic acid complex formation, Food Chemistry, 217, 9-17 (2017)

Bonilla, J., E. Talón y otros tres autores, Effect of the incorporation of antioxidants on physicochemical and antioxidant properties of wheat starch - chitosan films, Journal of Food Engineering, 118(3), 271-278 (2014)

Boom, E.A., J.A. Orozco, J.D. Alean y B. Rojano, Evaluación de la Actividad Antioxidante de Aceites Esenciales de Eucaliptos Cultivados en Colombia, Información Tecnologica, 29(6), 57-66 (2018)

Brand-Williams, W., M.E. Cuvelier y C. Berset, Use of a Free Radical Method to Evaluate Antioxidant Activity, Food Sci. Technol., 28, 25-30 (1995)

Carissimi, M., S.H. Flôres y R. Rech, Effect of Microalgae Addition on Active Biodegradable Starch Film, Algal Res., 32, 201-209 (2018)

Garavand, F., M. Rouhi y otros tres autores, Improving the integrity of natural biopolymer films used in food packaging by crosslinking approach: A review, International Journal of Biological Macromolecules, 104(June), 687-707 (2017)

Hoover, R., The Impact of Heat-Moisture Treatment on Molecular Structures and Properties of Starches Isolated from Different Botanical Sources, Critical Reviews in Food Science and Nutrition, 50(9), 835-847 (2010)

$\mathrm{Hu}, \mathrm{X}$., B. Guo y otros nueve autores, Modification of potato starch by using superheated steam, Carbohydrate Polymers, 198, 375-384 (2018)

Huang, S.H. y L.T. Ng, Quantification of polyphenolic content and bioactive constituents of some commercial rice varieties in Taiwan, Journal of Food Composition and Analysis, 26(1-2), 122-127 (2012)

ISO 12647-2, International Standard for the Production of Half-Tone Colour (2004)

López-Rubio, A. y J.M. Lagaron, Improvement of UV stability and mechanical properties of biopolyesters through the addition of $\beta$-carotene, Polym Degrad Stab, 95(11), 2162-2168 (2010)

Luna, G., H. Villada y R. Velasco, Almidón Termoplástico de Yuca Reforzado con Fibra de Fique: Preliminares, Dyna, 76(159), 145-151 (2010)

Mesa-Vanegas, A. M., C.A. Gaviria y otros cuatro autores, Actividad antioxidante y contenido de fenoles totales de algunas especies del género Calophyllum, Revista cubana de plantas medicinales, 15(2), 13-15 (2014)

Pastor, C., L. Sánchez-González y otros tres autores, Physical and antioxidant properties of chitosan and methylcellulose based films containing resveratrol, Food Hydrocoll., 30(1), 272-280 (2014)

Singleton, V.L., Colorimetry of Total Phenolics with Phosphomolybdic-Phosphotungstic Acid Reagents, Am J. Enol. Viticult., 16(3), 144-158 (1965)

Stoll, L., R. Rech y otros tres autores, Carotenoids extracts as natural colorants in poly(lactic acid) films, J. Appl Polym Sci., 135(33), 46585 (2018)

Talón, E., K.T. Trifkovic y otros cinco autores, Antioxidant Edible Films Based on Chitosan and Starch Containing Polyphenols from Thyme Extracts, Carbohydr. Polym., 157, 1153-1161 (2017)

Tanetrungroj, Y. y J. Prachayawarakorn, Effect of dual modification on properties of biodegradable crosslinked-oxidized starch and oxidized-crosslinked starch films, International Journal of Biological Macromolecules, 120, 1240-1246 (2018)

Tran, T.N., A. Athanassiou, A. Basit e I.S. Bayer, Starch-Based Bio-Elastomers Functionalized with Red Beetroot Natural Antioxidant. Food Chem., 216, 324-333 (2017)

Villada, H.S. H.A. Acosta y R.J. Velasco, Investigación de Almidones Termoplásticos, Precursores de Productos Biodegradables, Información Tecnológica 19(2), 3-14 (2008) 